\title{
ON THIRD ORDER HANKEL DETERMINANT FOR INVERSE FUNCTIONS OF CERTAIN CLASSES OF UNIVALENT FUNCTIONS
}

\author{
MILUTIN OBRADOVIĆ ${ }^{1}$, NIKOLA TUNESKI ${ }^{2, *}$
}

\begin{abstract}
In this paper we determine the upper bounds for the Hankel determinant of third order for the inverse functions of functions from some classes of univalent functions.
\end{abstract}

\section{INTRODUCTION AND PRELIMINARIES}

Let $\mathcal{A}$ be the class containing functions that are analytic in the unit disk $\mathbb{D}:=|z|<1$ and are normalized such that $f(0)=0=f^{\prime}(0)-1$, i.e.,

$$
f(z)=z+a_{2} z^{2}+a_{3} z^{3}+\cdots
$$

By $\mathcal{S}$ we denote the class of functions from $\mathcal{A}$ which are univalent in $\mathbb{D}$.

A problem that recently rediscovered, is to find upper bound (preferably sharp) of the modulus of the Hankel determinant $H_{q}(n)(f)$ of a given function $f$, for $q \geq 1$ and $n \geq 1$, defined by

$$
H_{q}(n)(f)=\left|\begin{array}{cccc}
a_{n} & a_{n+1} & \ldots & a_{n+q-1} \\
a_{n+1} & a_{n+2} & \ldots & a_{n+q} \\
\vdots & \vdots & & \vdots \\
a_{n+q-1} & a_{n+q} & \ldots & a_{n+2 q-2}
\end{array}\right| .
$$

The general Hankel determinant is hard to deal with, so the second and the third ones,

$$
H_{2}(2)(f)=\left|\begin{array}{ll}
a_{2} & a_{3} \\
a_{3} & a_{4}
\end{array}\right|=a_{2} a_{4}-a_{3}^{2}
$$

and

$$
H_{3}(1)(f)=\left|\begin{array}{ccc}
1 & a_{2} & a_{3} \\
a_{2} & a_{3} & a_{4} \\
a_{3} & a_{4} & a_{5}
\end{array}\right|=a_{3}\left(a_{2} a_{4}-a_{3}^{2}\right)-a_{4}\left(a_{4}-a_{2} a_{3}\right)+a_{5}\left(a_{3}-a_{2}^{2}\right),
$$

respectively, are studied instead. The research is focused on the subclasses of univalent functions (starlike, convex, $\alpha$-convex, close-to-convex, spirallike,...) since the general class of normalised

\footnotetext{
${ }^{1}$ Department of Mathematics, Faculty of Civil Engineering, University of Belgrade, Bulevar Kralja Aleksandra 73, 11000, Belgrade, Serbia

${ }^{2}$ Department of Mathematics and Informatics, Faculty of Mechanical Engineering, Ss. Cyril and Methodius University in Skopje, Karpoš II B.B., 1000 Skopje, Republic of North Macedonia

${ }^{*}$ CORRESPONDING AUTHOR

E-mail address: obrad@grf.bg.ac.rs, nikola.tuneski@mf.ukim.edu.mk.

Key words and phrases. Hankel determinant; third order; inverse functions; classes.

Received 09/11/2021.
} 
univalent functions is also hard to deal with. Some of the more significant results can be found in $[2-8,10]$.

In this paper we will give the upper bound of the modulus of the third Hankel determinant for the inverse functions for the functions in different subclasses of $\mathcal{S}$ as listed bellow.

The classes $\mathcal{R}, \mathcal{C}, \mathcal{S}^{\star}, \mathcal{S}_{s}^{\star}$ (with bounded turning, convex, starlike and starlike with respect to symmetric points, respectively) are defined in the following way:

$$
\begin{aligned}
\mathcal{R} & =\left[f \in \mathcal{A}: \operatorname{Re} f^{\prime}(z)>0, z \in \mathbb{D}\right], \\
\mathcal{C} & =\left[f \in \mathcal{A}: \operatorname{Re}\left(1+\frac{z f^{\prime \prime}(z)}{f^{\prime}(z)}\right)>0, z \in \mathbb{D}\right], \\
\mathcal{S}^{\star} & =\left[f \in \mathcal{A}: \operatorname{Re} \frac{z f^{\prime}(z)}{f(z)}>0, z \in \mathbb{D}\right], \\
\mathcal{S}_{s}^{\star} & =\left[f \in \mathcal{A}: \operatorname{Re} \frac{2 z f^{\prime}(z)}{f(z)-f(-z)}>0, z \in \mathbb{D}\right] .
\end{aligned}
$$

For every univalent function in $\mathbb{D}$, exists inverse at least on the disk with radius $1 / 4$ (due to the famous Koebe's $1 / 4$ theorem). If the inverse has an expansion

$$
f^{-1}(w)=w+A_{2} w^{2}+A_{3} w^{3}+\cdots
$$

then, by using the identity $f\left(f^{-1}(w)\right)=w$, from (1) and (2) we receive

$$
\begin{aligned}
& A_{2}=-a_{2}, \\
& A_{3}=-a_{3}+2 a_{2}^{2}, \\
& A_{4}=-a_{4}+5 a_{2} a_{3}-5 a_{2}^{3}, \\
& A_{5}=-a_{5}+6 a_{2} a_{4}-21 a_{2}^{2} a_{3}+3 a_{3}^{2}+14 a_{2}^{4} .
\end{aligned}
$$

As for the Hankel determinant of the third order for inverse functions, by using the definition of $H_{3}(1)(f)$ and the relations (3), after some calculations, we receive:

$$
\begin{aligned}
H_{3}(1)\left(f^{-1}\right) & =A_{3}\left(A_{2} A_{4}-A_{3}^{2}\right)-A_{4}\left(A_{4}-A_{2} A_{3}\right)+A_{5}\left(A_{3}-A_{2}^{2}\right) \\
& =a_{3}\left(a_{2} a_{4}-a_{3}^{2}\right)-a_{4}\left(a_{4}-a_{2} a_{3}\right)+a_{5}\left(a_{3}-a_{2}^{2}\right)-\left(a_{3}-a_{2}^{2}\right)^{3} \\
& =H_{3}(1)(f)-\left(a_{3}-a_{2}^{2}\right)^{3}
\end{aligned}
$$

i.e.,

$$
H_{3}(1)\left(f^{-1}\right)=H_{3}(1)(f)-\left(a_{3}-a_{2}^{2}\right)^{3}
$$

and also,

$$
\left|H_{3}(1)\left(f^{-1}\right)-H_{3}(1)(f)\right|=\left|a_{3}-a_{2}^{2}\right|^{3} .
$$

For our consideration we need the next lemmas.

Lemma 1 ( [1]). Let

$$
\omega(z)=c_{1} z+c_{2} z^{2}+\cdots
$$


be a Schwartz function, i.e., a function analytic in $\mathbb{D}, \omega(0)=0$ and $|\omega(z)|<1$. Then

$$
\begin{aligned}
& \left|c_{1}\right| \leq 1, \quad\left|c_{2}\right| \leq 1-\left|c_{1}\right|^{2}, \\
& \left|c_{3}\right| \leq 1-\left|c_{1}\right|^{2}-\frac{\left|c_{2}\right|^{2}}{1+\left|c_{1}\right|}, \quad \text { and } \quad\left|c_{4}\right| \leq 1-\left|c_{1}\right|^{2}-\left|c_{2}\right|^{2} .
\end{aligned}
$$

Lemma 2 ( [9]). Let $\omega$ given by (6) be a Schwartz function. Then, for any real numbers $\mu$ and $\nu$ from the sets $D_{1}$ and $D_{2}$, the following sharp estimate holds

$$
\left|c_{3}+\mu c_{1} c_{2}+\nu c_{1}^{3}\right| \leq 1
$$

where

$$
\begin{aligned}
& D_{1}=\left\{(\mu, \nu):|\mu| \leq \frac{1}{2},-1 \leq \nu \leq 1\right\}, \\
& D_{2}=\left\{(\mu, \nu): \frac{1}{2} \leq|\mu| \leq 2, \frac{4}{27}(|\mu|+1)^{3}-(|\mu|+1) \leq \nu \leq 1\right\} .
\end{aligned}
$$

\section{MAin Results}

Theorem 1. Let $f \in \mathcal{R}$. Then we have

$$
\left|H_{3}(1)\left(f^{-1}\right)\right| \leq 0.593155 \ldots
$$

Proof. Since $f \in \mathcal{R}$ is equivalent to

$$
f^{\prime}(z)=\frac{1+\omega(z)}{1-\omega(z)}
$$

where $\omega$ is a Schwartz function, then

$$
f^{\prime}(z)=1+2 \omega(z)+2 \omega^{2}(z)+\cdots .
$$

Using the notations for $f$ and $\omega$ given by (1) and (6) and equating the coefficients in (7), we have

$$
\left\{\begin{array}{l}
a_{2}=c_{1}, \\
a_{3}=\frac{2}{3}\left(c_{2}+c_{1}^{2}\right) \\
a_{4}=\frac{1}{2}\left(c_{3}+2 c_{1} c_{2}+c_{1}^{3}\right), \\
a_{5}=\frac{2}{5}\left(c_{4}+2 c_{1} c_{3}+3 c_{1}^{2} c_{2}+c_{2}^{2}+c_{1}^{4}\right) .
\end{array}\right.
$$

Now, from (4) and (8), and some computations, for $f \in \mathcal{R}$ we obtain:

$$
\begin{aligned}
H_{3}(1)\left(f^{-1}\right) & =\frac{1}{540}\left[-135 c_{3}^{2}+108 c_{1} c_{2} c_{3}-54 c_{1}^{3} c_{3}+300 c_{1}^{2} c_{2}^{2}\right. \\
& \left.-132 c_{1}^{4} c_{2}-176 c_{2}^{3}+13 c_{1}^{6}+72\left(2 c_{2}-c_{1}^{2}\right) c_{4}\right] .
\end{aligned}
$$

From (9), after some rearrangements we have

$$
\begin{aligned}
& 540 \cdot H_{3}(1)\left(f^{-1}\right) \\
= & -135 c_{3}\left(c_{3}+\frac{8}{45} c_{1} c_{2}+\frac{2}{5} c_{1}^{3}\right)+132 c_{1} c_{2}\left(c_{3}+\frac{1}{2} c_{1} c_{2}-c_{1}^{3}\right) \\
+ & 234 c_{1}^{2} c_{2}^{2}-176 c_{2}^{3}+13 c_{1}^{6}+72\left(2 c_{2}-c_{1}^{2}\right) c_{4}
\end{aligned}
$$


and from here

$$
\begin{aligned}
& 540\left|H_{3}(1)\left(f^{-1}\right)\right| \\
\leq & 135\left|c_{3}\right|\left|c_{3}+\frac{8}{45} c_{1} c_{2}+\frac{2}{5} c_{1}^{3}\right|+132\left|c_{1}\right|\left|c_{2}\right|\left|c_{3}+\frac{1}{2} c_{1} c_{2}-c_{1}^{3}\right| \\
+ & 234\left|c_{1}\right|^{2}\left|c_{2}\right|^{2}+176\left|c_{2}\right|^{3}+13\left|c_{1}\right|^{6}+72\left(2\left|c_{2}\right|+\left|c_{1}\right|^{2}\right)\left|c_{4}\right| .
\end{aligned}
$$

Using the results of Lemma 2 (case $\left.D_{1}\right)$ and Lemma 1 for $\left|c_{4}\right|$, from the last relation we have

$$
\begin{aligned}
& 540\left|H_{3}(1)\left(f^{-1}\right)\right| \\
\leq & 135\left|c_{3}\right|+132\left|c_{1}\right|\left|c_{2}\right|+234\left|c_{1}\right|^{2}\left|c_{2}\right|^{2}+176\left|c_{2}\right|^{3}+13\left|c_{1}\right|^{6} \\
& +72\left(2\left|c_{2}\right|+\left|c_{1}\right|^{2}\right)\left(1-\left|c_{1}\right|^{2}-\left|c_{2}\right|^{2}\right) \\
= & 135\left|c_{3}\right|+132\left|c_{1}\right|\left|c_{2}\right|+176\left|c_{1}\right|^{2}\left|c_{2}\right|^{2}+32\left|c_{2}\right|^{3}+13\left|c_{1}\right|^{6} \\
& +144\left|c_{2}\right|-144\left|c_{1}\right|^{2}\left|c_{2}\right|+72\left|c_{1}\right|^{2}-72\left|c_{1}\right|^{4}
\end{aligned}
$$

Using Lemma 2 we have next estimation for $\left|c_{3}\right|$ :

$$
\begin{aligned}
\left|c_{3}\right| & \leq 1-\left|c_{1}\right|^{2}-\frac{\left|c_{2}\right|^{2}}{1+\left|c_{1}\right|} \\
& =1-\left|c_{1}\right|^{2}-\left|c_{2}\right|^{2}+\frac{\left|c_{1}\right|\left|c_{2}\right|^{2}}{1+\left|c_{1}\right|} \\
& \leq 1-\left|c_{1}\right|^{2}-\left|c_{2}\right|^{2}+\frac{\left|c_{1}\right|}{1+\left|c_{1}\right|}\left(1-\left|c_{1}\right|^{2}\right)^{2}
\end{aligned}
$$

i.e.,

$$
\left|c_{3}\right| \leq\left(1-\left|c_{1}\right|^{2}\right)^{2}+\left|c_{1}\right|\left(1-\left|c_{1}\right|^{2}\right)-\left|c_{2}\right|^{2}
$$

If we use the estimation given in (10) in the last relation for $540\left|H_{3}(1)\left(f^{-1}\right)\right|$, then we have

$$
\begin{aligned}
540\left|H_{3}(1)\left(f^{-1}\right)\right| \leq & 135\left(1-\left|c_{1}\right|^{2}\right)^{2}+135\left|c_{1}\right|\left(1-\left|c_{1}\right|^{2}\right)-135\left|c_{2}\right|^{2} \\
& +132\left|c_{1}\right|\left|c_{2}\right|+176\left|c_{1}\right|^{2}\left|c_{2}\right|^{2}+32\left|c_{2}\right|^{3}+13\left|c_{1}\right|^{6} \\
& +144\left|c_{2}\right|-144\left|c_{1}\right|^{2}\left|c_{2}\right|+72\left|c_{1}\right|^{2}-72\left|c_{1}\right|^{4} \\
= & 135\left(1-\left|c_{1}\right|^{2}\right)^{2}+135\left|c_{1}\right|\left(1-\left|c_{1}\right|^{2}\right)+132\left|c_{1}\right|\left|c_{2}\right| \\
& +176\left|c_{1}\right|^{2}\left|c_{2}\right|^{2}+13\left|c_{1}\right|^{6}+144\left|c_{2}\right|-144\left|c_{1}\right|^{2}\left|c_{2}\right| \\
& +72\left|c_{1}\right|^{2}-72\left|c_{1}\right|^{4}-\left|c_{2}\right|^{2}\left(135-32\left|c_{2}\right|\right)
\end{aligned}
$$

Finally, using the estimation $\left|c_{2}\right| \leq 1-\left|c_{1}\right|^{2}$ and some calculations, from the last relation we obtain that

$$
\begin{aligned}
540\left|H_{3}(1)\left(f^{-1}\right)\right| & \leq 175\left|c_{1}\right|^{6}-117\left|c_{1}\right|^{4}-267\left|c_{1}\right|^{3}-324\left|c_{1}\right|^{2}+267\left|c_{1}\right|+279 \\
& \leq 320.30 \ldots,
\end{aligned}
$$

since the function on the right hand side of $\left|c_{1}\right|$ has maximum value $320.30 \ldots$ in the interval $[0,1]$, obtained for $\left|c_{1}\right|=0.23887 \ldots$ So, for $f \in \mathcal{R}$ :

$$
\left|H_{3}(1)\left(f^{-1}\right)\right| \leq \frac{320.30 \ldots}{540}=0.59315 \ldots
$$


Remark 1. Since for $f \in \mathcal{R}$, by using the relation (8), we have

$$
\left|a_{3}-a_{2}^{2}\right|^{3}=\left|\frac{2}{3}\left(c_{2}+c_{1}^{2}\right)-c_{1}^{2}\right|^{3} \leq \frac{1}{27}\left(2\left|c_{2}\right|+\left|c_{1}\right|^{2}\right)^{3} \leq \frac{1}{27}\left(2-\left|c_{1}\right|^{2}\right)^{3} \leq \frac{8}{27},
$$

where the equality attains attains for $\left|c_{1}\right|=0$ and $\left|c_{2}\right|=1$. In that sense, from (5) we obtain

$$
\left|H_{3}(1)\left(f^{-1}\right)-H_{3}(1)(f)\right| \leq \frac{8}{27},
$$

and the result is sharp. The extremal function is defined by $f^{\prime}(z)=\frac{1+z^{2}}{1-z^{2}}$, i.e., by $f(z)=$ $-z+\ln \frac{1+z}{1-z}$.

Theorem 2. Let $f \in \mathcal{C}$, then

$$
\left|H_{3}(1)\left(f^{-1}\right)\right| \leq \frac{11}{180}=0.061 \ldots
$$

Proof. From the definition of the class $\mathcal{C}$ we have

$$
1+\frac{z f^{\prime \prime}(z)}{f^{\prime}(z)}=\frac{1+\omega(z)}{1-\omega(z)}
$$

where $\omega$ is a Schwartz function, and from here

$$
\left(z f^{\prime}(z)\right)^{\prime}=\left[1+2\left(\omega(z)+\omega^{2}(z)+\cdots\right)\right] \cdot f^{\prime}(z) .
$$

Using the notations (1) and (6), and comparing the coefficients in the relation (12), then, after some simple calculations, we obtain

$$
\left\{\begin{array}{l}
a_{2}=c_{1}, \\
a_{3}=\frac{1}{3}\left(c_{2}+3 c_{1}^{2}\right), \\
a_{4}=\frac{1}{6}\left(c_{3}+5 c_{1} c_{2}+6 c_{1}^{3}\right) \\
a_{5}=\frac{1}{30}\left(3 c_{4}+14 c_{1} c_{3}+43 c_{1}^{2} c_{2}+30 c_{1}^{4}+6 c_{2}^{2}\right) .
\end{array}\right.
$$

From the relations (4) and (13), and some transformations, we get

$$
H_{3}(1)\left(f^{-1}\right)=\frac{1}{540}\left(-6 c_{1} c_{2} c_{3}+3 c_{1}^{2} c_{2}^{2}-4 c_{2}^{3}-15 c_{3}^{2}+18 c_{2} c_{4}\right) .
$$

From the previous relation we have

$$
540 \cdot H_{3}(1)\left(f^{-1}\right)=-15\left(c_{3}+\frac{1}{5} c_{1} c_{2}\right)^{2}+\frac{18}{5} c_{1}^{2} c_{2}^{2}-4 c_{2}^{3}+18 c_{2} c_{4},
$$

and from here

$$
\begin{aligned}
540\left|H_{3}(1)\left(f^{-1}\right)\right| & \leq 15\left|c_{3}+\frac{1}{5} c_{1} c_{2}\right|^{2}+\frac{18}{5}\left|c_{1}\right|^{2}\left|c_{2}\right|^{2}+4\left|c_{2}\right|^{3}+18\left|c_{2}\right|\left|c_{4}\right| \\
& \leq 15+\frac{18}{5}\left|c_{1}\right|^{2}\left|c_{2}\right|^{2}+4\left|c_{2}\right|^{3}+18\left|c_{2}\right|\left(1-\left|c_{1}\right|^{2}-\left|c_{2}\right|^{2}\right) \\
& =15+18\left|c_{2}\right|-\frac{18}{5}\left|c_{1}\right|^{2}\left|c_{2}\right|\left(5-\left|c_{2}\right|\right)-14\left|c_{2}\right|^{3} \\
& \leq 15+18\left|c_{2}\right| \\
& \leq 33
\end{aligned}
$$

where we used Lemma 1 and Lemma 2. From the previous relations we have the statement of the theorem. 
Remark 2. In the case when $f \in \mathcal{C}$, by using the relation (13), we have

$$
\left|a_{3}-a_{2}^{2}\right|^{3}=\frac{1}{27}\left|c_{2}\right|^{3} \leq \frac{1}{27}
$$

with equality for $\left|c_{2}\right|=1$. This, using (5), implies that

$$
\left|H_{3}(1)\left(f^{-1}\right)-H_{3}(1)(f)\right| \leq \frac{1}{27},
$$

with sharpness for the function defined by (11) and $\omega(z)=z^{2}$.

Theorem 3. Let $f \in \mathcal{S}^{\star}$. Then

$$
\left|H_{3}(1)\left(f^{-1}\right)-H_{3}(1)(f)\right| \leq 1
$$

with equality sign for the Koebe function, and

$$
\left|H_{3}(1)\left(f^{-1}\right)\right|<\frac{14}{9}=1.555 \ldots
$$

Proof. For $f \in \mathcal{S}^{\star}$ we have $\left|a_{3}-a_{2}^{2}\right| \leq 1$ (as for the class $\mathcal{S}$ ), so that from (5):

$$
\left|H_{3}(1)\left(f^{-1}\right)-H_{3}(1)(f)\right| \leq 1 \text {. }
$$

Further, using that for $f \in \mathcal{S}^{\star},\left|H_{3}(1)(f)\right|<\frac{5}{9}$ ( [11, Theorem 1]), we have

$$
\left|H_{3}(1)\left(f^{-1}\right)\right| \leq 1+\left|H_{3}(1)(f)\right|<1+\frac{5}{9}=\frac{14}{5} \text {. }
$$

Theorem 4. Let $f \in \mathcal{S}_{s}^{\star}$. Then

$$
\left|H_{3}(1)\left(f^{-1}\right)\right| \leq \frac{5}{4}
$$

Proof. Using the definition of the class $\mathcal{S}_{s}^{\star}$ we have that there exists a Schwartz function $\omega$ such that

$$
\frac{2 z f^{\prime}(z)}{f(z)-f(-z)}=\frac{1+\omega(z)}{1-\omega(z)}
$$

and from here

$$
2 z f^{\prime}(z)=\left[1+2\left(\omega(z)+\omega^{2}(z)+\cdots\right)\right] \cdot[f(z)-f(-z)] .
$$

Similarly, as in two previous cases (in Theorem 1 and Theorem 2), by comparing the coefficients in the relation (15), after some simple calculations, we have

$$
\left\{\begin{array}{l}
a_{2}=c_{1} \\
a_{3}=c_{2}+c_{1}^{2} \\
a_{4}=\frac{1}{2}\left(c_{3}+3 c_{1} c_{2}+2 c_{1}^{3}\right) \\
a_{5}=\frac{1}{2}\left(c_{4}+2 c_{1} c_{3}+5 c_{1}^{2} c_{2}+2 c_{1}^{4}+2 c_{2}^{2}\right) .
\end{array}\right.
$$

Now,from (4) and (16) and some calculations, for $f \in \mathcal{S}_{s}^{\star}$ we obtain:

$$
\begin{aligned}
H_{3}(1)\left(f^{-1}\right) & =\frac{1}{4}\left(-c_{3}^{2}+2 c_{1} c_{2} c_{3}+c_{1}^{2} c_{2}^{2}-4 c_{2}^{3}+2 c_{2} c_{4}\right) \\
& =\frac{1}{4}\left[-\left(c_{3}-c_{1} c_{2}\right)^{2}+2 c_{1}^{2} c_{2}^{2}-4 c_{2}^{3}+2 c_{2} c_{4}\right],
\end{aligned}
$$


which implies

$$
\left|H_{3}(1)\left(f^{-1}\right)\right| \leq \frac{1}{4}\left(\left|c_{3}-c_{1} c_{2}\right|^{2}+2\left|c_{1}\right|^{2}\left|c_{2}\right|^{2}+4\left|c_{2}\right|^{3}+2\left|c_{2}\right|\left|c_{4}\right|\right) .
$$

From the last relation and Lemma $2\left(\right.$ case $\left.D_{2}\right)$ and Lemma 1, we have:

$$
\begin{aligned}
\left|H_{3}(1)\left(f^{-1}\right)\right| & \leq \frac{1}{4}\left[1+2\left|c_{1}\right|^{2}\left|c_{2}\right|^{2}+4\left|c_{2}\right|^{3}+2\left|c_{2}\right|\left(1-\left|c_{1}\right|^{2}-\left|c_{2}\right|^{2}\right)\right] \\
& =\frac{1}{4}\left[1+2\left|c_{2}\right|+2\left|c_{2}\right|^{3}-2\left|c_{1}\right|^{2}\left|c_{2}\right|\left(1-\left|c_{2}\right|\right)\right] \\
& \leq \frac{1}{4}\left(1+2\left|c_{2}\right|+2\left|c_{2}\right|^{3}\right) \\
& \leq \frac{5}{4}
\end{aligned}
$$

Remark 3. For $f \in \mathcal{S}_{s}^{\star}$ we have $\left|a_{3}-a_{2}^{2}\right|^{3}=\left|c_{2}\right|^{3} \leq 1$, so that from (5):

$$
\left|H_{3}(1)\left(f^{-1}\right)-H_{3}(1)(f)\right| \leq 1 \text {. }
$$

The equality is attained for the function $f$ defined by

$$
\frac{2 z f^{\prime}(z)}{f(z)-f(-z)}=\frac{1+z^{2}}{1-z^{2}}
$$

i.e., with $\omega(z)=z^{2}$ in (14).

\section{REFERENCES}

[1] F. Carlson, Sur les coefficients d'une fonction bornée dans le cercle unité, Ark. Mat. Astr. Fys. 27A (1) (1940) 8.

[2] N.E. Cho, B. Kowalczyk, O.S. Kwon, A. Lecko, Y.J. Sim, Some coefficient inequalities related to the Hankel determinant for strongly starlike functions of order alpha, J. Math. Inequal. 11 (2) (2017) 429-439. https://doi.org/10.7153/jmi-11-36.

[3] W.K. Hayman, On the second Hankel determinant of mean univalent functions, Proc. London Math. Soc. (3) 18 (1068) 77-94.

[4] A. Janteng, S.A. Halim, M. Darus, Hankel determinant for starlike and convex functions, Int. J. Math. Anal. 1 (13) (2007) 619-625.

[5] S.K. Lee, V. Ravichandran, S. Supramaniam, Bounds for the second Hankel determinant of certain univalent functions, J. Inequal Appl. 2013 (2013) 281. https://doi.org/10.1186/1029-242X-2013-281

[6] M. Obradović, N. Tuneski, Some properties of the class $\mathcal{U}$, Ann. Univ. Mariae Curie-Skłodowska. Sect. A - Math. 73 (1) (2019) 49-56. https://doi.org/10.17951/a.2019.73.1.49-56.

[7] M. Obradović, N. Tuneski, Hankel determinant of second order for inverse functions of certain classes of univalent functions, submitted.

[8] M. Obradović, N. Tuneski, Hankel determinants of second and third order for the inverse of an univalent function, submitted.

[9] D.V. Prokhorov, J. Szynal, Inverse coefficients for $(\alpha, \beta)$-convex functions, Ann. Univ. Mariae CurieSkłodowska Sect. A 35 (1981) (1984) 125-143.

[10] P. Zaprawa, Third Hankel determinants for subclasses of univalent functions, Mediterr. J. Math. 14 (2017) 19. https://doi.org/10.1007/s00009-016-0829-y.

[11] P. Zaprawa, M. Obradovic, N. Tuneski, Third Hankel determinant for the class of univalent starlike functions, Rev. R. Acad. Cienc. Exactas Fís. Nat., Ser. A Mat. 115 (2021) 49. 